\title{
Virtual Reality-Guided Motor Imagery Increases Corticomotor Excitability in Healthy Volunteers and Stroke Patients
}

\author{
Hyungjun Im, MD ${ }^{1}$ Jeunghun Ku, PhD ${ }^{2}$, Hyun Jung Kim, $\mathrm{MD}^{1}$, Youn Joo Kang, $\mathrm{MD}^{1}$ \\ 'Department of Rehabilitation Medicine, Eulji Hospital, Eulji University School of Medicine, Seoul; \\ ${ }^{2}$ Department of Biomedical Engineering, Keimyung University, Daegu, Korea
}

Objective To investigate the effects of using motor imagery (MI) in combination with a virtual reality (VR) program on healthy volunteers and stroke patients. In addition, this study investigated whether task variability within the VR-guided MI programs would influence corticomotor excitability.

Methods The present study included 15 stroke patients and 15 healthy right-handed volunteers who were presented with four different conditions in a random order: rest, MI alone, VR-guided MI, and VR-guided MI with task variability. The corticomotor excitability of each participant was assessed before, during, and after each condition by measuring changes in the various parameters of motor-evoked potentials (MEPs) of the extensor carpi radials (ECR). Changes in intracortical inhibition (ICI) and intracortical facilitation (ICF) were calculated after each condition as percentages of inhibition (\%INH) and facilitation (\%FAC) at rest.

Results In both groups, the increases in MEP amplitudes were greater during the two VR-guided MI conditions than during MI alone. Additionally, the reductions in ECR \% INH in both groups were greater under the condition involving VR-guided MI with task variability than under that involving VR-guided MI with regular interval.

Conclusion The corticomotor excitability elicited by MI using a VR avatar representation was greater than that elicited by MI with real body observations. Furthermore, the use of task variability in a VR program may enhance neural regeneration after stroke by reducing ICI. The present findings support the use of various VR programs as well as the concept of combining MI with VR programs for neurorehabilitation.

Keywords Imagery (psychotherapy), Stroke, Transcranial magnetic stimulation, Upper extremity, Virtual reality exposure therapy

Department of Rehabilitation Medicine, Eulji Hospital, Eulji University School of Medicine, 68 Hangeulbiseok-ro, Nowon-gu, Seoul 01830, Korea. Tel: +82-2-970-8315, Fax: +82-2-979-8268, E-mail: md52516@hanmail.net

ORCID: Hyungjun Im (http://orcid.org/0000-0002-2360-7775); Jeunghun Ku (http://orcid.org/0000-0002-9610-0078); Hyun Jung Kim (http://orcid. org/0000-0002-2198-5668); Youn Joo Kang (http://orcid.org/0000-0002-9938-5435).

() This is an open-access article distributed under the terms of the Creative Commons Attribution Non-Commercial License (http://creativecommons.org/ licenses/by-nc/4.0) which permits unrestricted noncommercial use, distribution, and reproduction in any medium, provided the original work is properly cited. Copyright $\odot 2016$ by Korean Academy of Rehabilitation Medicine 


\section{INTRODUCTION}

Because long-term impairments of the upper extremities influence the ability of approximately $65 \%$ of patients to perform the activities of daily living following a stroke, exercise programs aimed at restoring function in the upper extremities are an important part of stroke rehabilitation [1]. However, a recent Cochrane review reported that despite the existence of a number of moderate-quality interventions, including virtual reality (VR), mental practice/motor imagery (MI), constraint-induced movement therapy, high doses of repetitive task practice, mirror therapy, and interventions for sensory impairment, there are currently no high-quality evidences for interventions in the field of upper extremity rehabilitation for stroke patients [2].

The observation of other individuals performing skilled movements as well as MI are effective for motor training [3]. Neuroimaging studies have shown that the primary motor cortex (M1) and secondary motor areas, including the premotor cortex, supplementary motor area, and the parietal cortices, are activated during MI tasks and motor execution [4]. Furthermore, recent randomized clinical trials evaluating the effectiveness of MI for the improvement of upper extremity motor dysfunction have returned promising results [5]. VR training is also an emerging technology in the field of rehabilitation. Recent randomized controlled trials with large sample sizes that utilized VR training for the upper extremities after stroke have tended to focus on various sensorimotor feedback and augmented reality technologies and have provided encouraging findings $[6,7]$. Furthermore, functional magnetic resonance imaging (fMRI) studies have demonstrated the occurrence of visuomotor cortical facilitation during VR training $[8,9]$. Relative to conventional therapies for stroke patients, VR training has many advantages. For example, because it provides a goal-directed task for the patient, VR training is more effective and intensive than self-training, and may also boost the motivation of patients and serve as a pleasurable experience during treatment by controlling the level of difficulty and the variability of the task $[10,11]$.

Because MI and VR applications are increasingly emerging as potentially useful techniques for rehabilitation after stroke, the present study was designed to determine whether MI combined with a VR program would have synergistic effects on the patient and provide superior corticomotor facilitation, as compared with MI alone. Thus, we first assessed stroke patients and healthy volunteers to investigate the combined effects of $\mathrm{MI}$ and VR training. Second, we investigated whether task variability within the VR-guided MI programs would influence corticomotor excitability or intracortical inhibition (ICI).

It is possible to evaluate corticomotor excitability by applying single- and paired-pulse transcranial magnetic stimulation (TMS). A decreased ICI in the early stages of stroke enhances neural plasticity $[12,13]$. The assessment of the parameters of motor-evoked potentials (MEPs), such as resting motor threshold (RMT), amplitude, area, ICI, and intracortical facilitation (ICF), allows for the identification of differences in corticomotor facilitation under various experimental conditions.

\section{MATERIALS AND METHODS}

\section{Participants}

The present study included 15 right-handed healthy volunteers (12 males and 3 females; mean age, $31.73 \pm 6.22$ years) and 15 stroke patients (9 males and 6 females; mean age, $58.87 \pm 10.07$ years). Detailed data regarding age, gender, and handedness were provided in Table 1 . Although the mean age of the two groups differed significantly in the present study, a previous study found that the ability to exhibit MI-induced corticomotor facilitation appears to be largely preserved throughout aging [14].

The healthy volunteers had no history of neurological or psychological disorders and no abnormalities were observed during their physical, neurological, and musculoskeletal examinations. According to the Edinburgh Handedness Inventory [15], all healthy volunteers were right-handed with a mean laterality quotient (LQ) of $84.77 \pm 17.25$. Additionally, none of the volunteers had any contraindications for TMS, such as intracranial metallic pieces or cardiac pacemakers [16]. The stroke group included patients with a first-ever stroke who were diagnosed by MRI or computed tomography scans, had mild to moderate hemiparesis of an upper extremity with a Medical Research Council (MRC) grade $\geq 3$ during the motor examination of the contralesional wrist extension, and had no upper extremity injuries or deformities. All subjects were able to sit upright throughout the experi- 
Hyungjun Im, et al.

Table 1. Demographic and baseline characteristics of subjects

\begin{tabular}{|c|c|c|c|c|c|c|}
\hline Patient no. & Sex & Age (yr) & Weeks since onset & Etiology & Site of lesion & VMIQ-2 \\
\hline 1 & M & 63 & 6 & Infarction & Rt. med. medullary & 1.75 \\
\hline 2 & $\mathrm{~F}$ & 62 & 3 & Infarction & $\begin{array}{l}\text { Rt. MCA } \\
\text { (cortical and subcortical) }\end{array}$ & 2.25 \\
\hline 3 & $\mathrm{~F}$ & 67 & 7 & Infarction & $\begin{array}{l}\text { Rt. MCA } \\
\text { (cortical and subcortical) }\end{array}$ & 2.00 \\
\hline 4 & $\mathrm{~F}$ & 68 & 7 & Hemorrhage & $\begin{array}{l}\text { Lt. thalamus (subcortical), } \\
\text { IVH }\end{array}$ & 2.00 \\
\hline 5 & M & 71 & 4 & Infarction & Rt. lat. medullary & 2.25 \\
\hline 6 & M & 54 & 4 & Infarction & Lt. pontine & 1.83 \\
\hline 7 & $\mathrm{~F}$ & 53 & 5 & Infarction & Rt. MCA (subcortical) & 1.67 \\
\hline 8 & M & 74 & 3 & Infarction & $\begin{array}{l}\text { Lt. PCA MCA ACA } \\
\text { (cortical and subcortical) }\end{array}$ & 1.83 \\
\hline 9 & M & 52 & 1 & Infarction & Lt. MCA (subcortical) & 2.00 \\
\hline 10 & $\mathrm{~F}$ & 59 & 4 & Infarction & Rt. pontine & 2.25 \\
\hline 11 & M & 35 & 3 & Infarction & $\begin{array}{l}\text { Lt. MCA } \\
\text { (cortical and subcortical) }\end{array}$ & 1.67 \\
\hline 12 & $\mathrm{~F}$ & 48 & 6 & Hemorrhage & Lt. BG & 2.25 \\
\hline 13 & M & 52 & 33 & Hemorrhage & Rt. post. medullary & 2.25 \\
\hline 14 & M & 62 & 1 & Infarction & $\begin{array}{l}\text { Rt. MCA } \\
\text { (cortical and subcortical) }\end{array}$ & 1.89 \\
\hline 15 & $\mathrm{~F}$ & 63 & 1 & Infarction & Lt. MCA (subcortical) & 2.17 \\
\hline
\end{tabular}

VMIQ-2, Vividness of Movement Imagery Questionnaire-2; MCA, middle cerebral artery; PCA, posterior cerebral artery; ACA, anterior cerebral artery; IVH, intraventricular hematoma; BG, basal ganglia.

ments and did not have any severe cognitive deficits.

The exclusion criteria for the present study were as follows: (1) severe motor weakness (MRC grade of wrist extension $\leq 2)$; (2) the absence of MEPs in the affected extensor carpi radialis (ECR) muscle; (3) severe depression, apraxia, and/or cognitive deficits with a score $<24$ on the Mini-Mental State Examination [17]; (4) a history of seizures; (5) the inability to perform MI using the Vividness of Movement Imagery Questionnaire-2 (VMIQ-2) based on an average score $\geq 4$ (vague and $\mathrm{dim}$ ) [18]; and/ or (6) any contraindications for TMS, such as intracranial metallic implants or cardiac pacemakers [16].

The Institutional Review Board of Eulji Hospital approved the protocol, and all subjects provided written informed consent. Following the experiment, all subjects were assessed to determine if there were any adverse effects from the TMS including dizziness, headaches, or neck pains.

\section{Experimental conditions}

The present study included four different conditions that were administered in a random order on the same day: rest (Condition A), MI alone (Condition B), VRguided MI (Condition C), and VR-guided MI with task variability (Condition $\mathrm{D}$ ). The subjects were asked to sit in a relaxed position during the performance of each condition, and more than 30 minutes of rest was allowed between the performances. Wrist exercise imagery was chosen because this type of movement plays an important functional role in the recovery of the upper extremity after stroke.

Under Condition A, the subjects were asked to watch a black screen without having other thoughts. Under Condition $\mathrm{B}$, the subjects were asked to imagine wrist extension while observing their own wrist; a metronome (20 beats per minute) was used to cue the MI. Under Condition $\mathrm{C}$, the extension of the wrist was imagined at a constant pace while observing an avatar on the monitor screen jump over obstacles at regular time intervals (5 seconds). Under Condition D, the subjects were asked to imagine wrist extension while an avatar on the screen jumped over obstacles at irregular time intervals (ran- 
domly between 3 and 7 seconds). During the performance of each condition, the experimenter instructed the subject not to make any voluntary movements, and muscle activation was monitored by electromyography (EMG) while performing MI.

\section{VR wrist program for MI}

\section{Set-up}

The VR wrist program for MI was developed by biomedical engineers, software engineers, and clinicians. The software was operated using a personal computer (PC) with a 19-inch monitor and a resolution of $1280 \times 1024$ in Windows 7 (Microsoft, Redmond, WA, USA). All subjects were seated in a comfortable chair with a headrest to secure the head position. The right forearm of the healthy volunteers and the affected forearm of the stroke patients were placed in a pronated position with their elbows flexed and secured at $90^{\circ}$ over an elbow rest on a desk (Fig. 1). The experimenter inspected and confirmed that there was no voluntary upper extremity movement during the experiments.

\section{Description of the VR program}

To elicit the effective MI, we provide specific experience within 3D VR simulation which attract user's attention using character, goal directed tasks (avatar running along a track and jumping over obstacles) and vivid acoustic

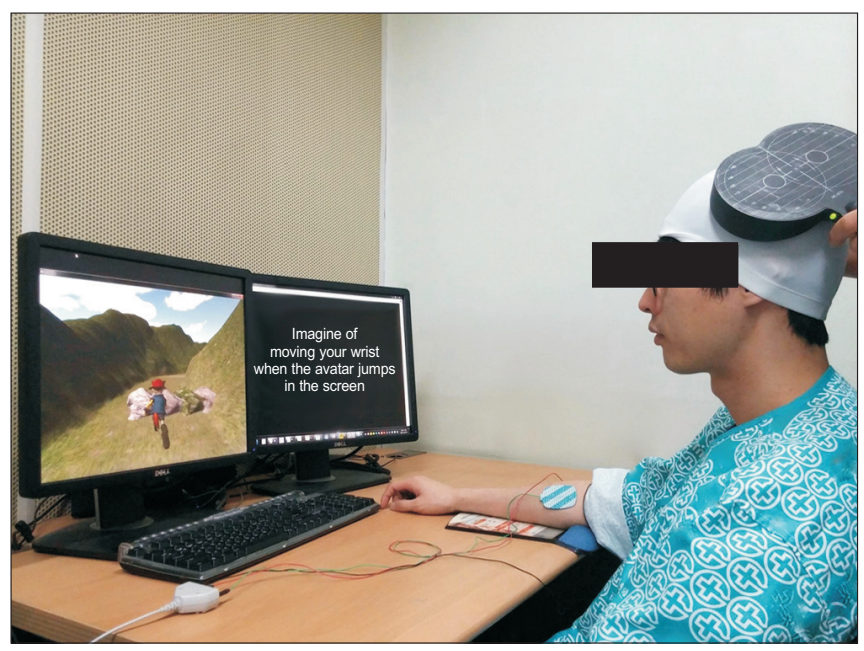

Fig. 1. The subjects were required to imagine wrist extension when the avatar jumped over obstacles in a virtual reality-guided motor imagery program. During the motor imagery, motor-evoked potentials were recorded. sound. The VR program for MI was composed of an avatar running along a track and jumping over obstacles; the intervals at which the obstacles would appear could be selected by the experimenter and could appear at either regular or irregular intervals in a variable manner. With this program, the subjects could recognize the moment that the character would have to jump or keep running. They were asked to imagine the performance of a wrist extension as the avatar jumped and maintained MI while the avatar was staying in the air (Fig. 1). The subjects listened to sound of foot step and jerk associated with running or jumping action.

\section{Transcranial magnetic stimulation}

The TMS was applied with a butterfly figure-of-eight coil (MC-B70; diameter, $97 \mathrm{~mm}$ ) attached to a MagPro R30 stimulator (MagVenture, Farum, Denmark). The MEPs were recorded from the contralateral ECR using the Dantec Keypoint EMG/NCS/EP Workstation (Alpine Biomed ApS, Skovlunde, Denmark). TMS was applied at the hot spot, which was the dominant M1 for the healthy volunteers and the ipsilesional M1 for the stroke patients, and the coil position was adjusted before starting the next condition. The coil was placed with the handle at a $45^{\circ}$ posterolaterally with respect to the mid sagittal axis of head by the same experimenter, and the hot spot was marked on a close-fitting cap with a 1-cm grid to ensure that the coil could be maintained at a constant position and checked by the experimenter throughout the procedure. The MEPs were recorded by a surface disc using $\mathrm{Ag} / \mathrm{AgCl}$ electrodes (diameter, $20 \mathrm{~mm}$ ) with the active electrode placed on the motor point of the ECR and the reference electrode attached to the tendon of the ECR muscle. The EMG signals were amplified and filtered (10 $\mathrm{Hz}$ to $1 \mathrm{kHz}$ ) and then sampled at $5 \mathrm{kHz}$.

The RMT was defined as the lowest current that evoked MEPs with a peak-to-peak amplitude greater than $50 \mu \mathrm{V}$ for at least four of eight consecutive stimuli. Subsequently, the test stimulus intensity was set to $120 \%$ of the RMT for each subject. The corticomotor excitability of each subject was compared before, during, and after each of the different conditions by assessing changes in the MEP amplitude $(\mathrm{mV})$, MEP negative area $(\mathrm{mV} / \mathrm{ms})$, ICI, and ICF (Fig. 2). When testing the paired-pulse paradigm, the conditioning stimulus intensity was set to $80 \%$ of the RMT and the inter-simulation interval (ISI) was set at 2 


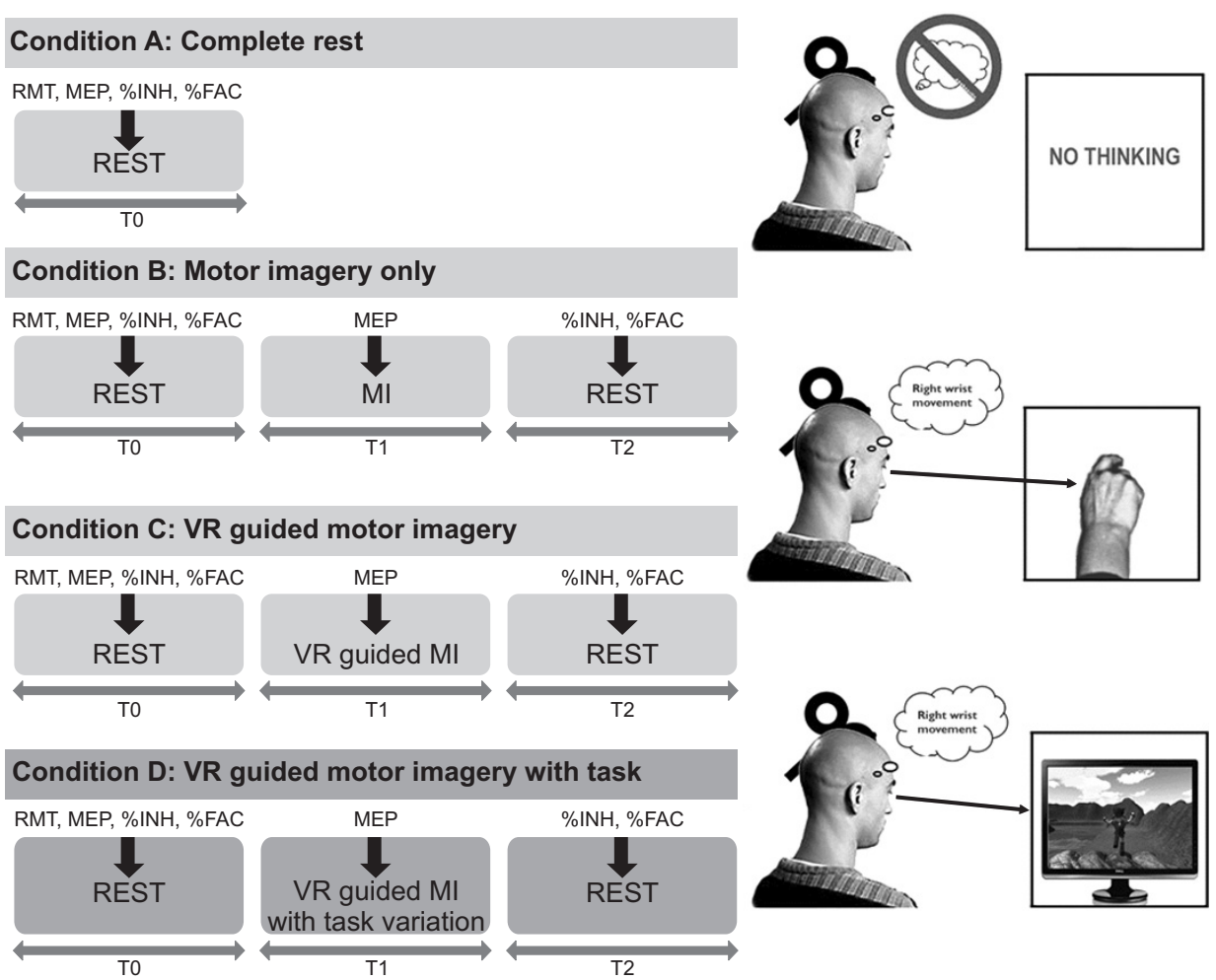

Fig. 2. Transcranial magnetic stimulation (TMS) was applied under four conditions and at various time points. Ten TMS applications was administered, and the MEPs were measured prior to, during, and after MI under each condition (B, C, and D; thick arrows). Under Condition A, the baseline MEP parameters were measured during complete rest. MEP, motor-evoked potential; RMT, resting motor threshold; \%INH, percent inhibition; \%FAC, percent facilitation; MI, motor imagery; VR, virtual reality. $\mathrm{ms}$ for ICI and $15 \mathrm{~ms}$ for ICF to allow for maximal inhibition and facilitation. ICI and ICF were determined based on the relationship between the sizes of the conditioned MEPs. TMS was delivered at intervals of at least 5 seconds and 10 MEPs were recorded per parameter under each of the four experimental conditions.

Measurements were taken at baseline (T0), during MI (T1), and post-MI (T2) during Conditions B, C, and D. The RMT, peak-to-peak MEP amplitude, negative MEP area, ICI, and ICF were recorded at baseline (T0), and the MEP amplitude and MEP area were measured during MI in wrist extension phase (T1). Finally, to assess changes in ICI and ICF, these variables were recorded immediately after the MI was finished (T2) and calculated as percentages of inhibition (\%INH) and facilitation (\%FAC) from baseline (T0). The order of stimulation for each MEP parameter was randomized. The time points for the TMS applications were shown in Fig. 2.

To assess peripheral nerve excitability, the supramaximal M waves (Max) of the median nerve of the wrist were evaluated after the performance of each condition. A 1-ms circular electrical stimulus was applied to the median nerve between the palmaris longus and the flexor carpi radialis tendons, and the resulting Max were recorded from the abductor pollicis brevis (APB) using paired $\mathrm{Ag}$ / $\mathrm{AgCl}$ disc electrodes (diameter, $20 \mathrm{~mm}$ ) that were placed on the center of the APB muscle belly and tendon after preparing the skin.

\section{Assessment of vividness of movement imagery}

The MI ability of the subjects was measured using the VMIQ-2 [18], a 12-item questionnaire that brings certain images to mind and requires the subject to rate them using a five-point scale according to the degree of clearness and vividness. A score of 1 represents a perfectly vivid image, as in normal vision, whereas a score of 5 represents no image at all. During the assessment, three types of MI are performed per item while the eyes are closed: (1) external visual imagery, or watching oneself perform the movement; (2) internal visual imagery, or looking through one's own eyes while performing the movement; and (3) kinesthetic imagery, or feeling oneself do the movement.

\section{Data analysis}

Ten MEPs were recorded per parameter (peak-to-peak amplitude, negative area the MEPs, ICI, and ICF) under each condition and were then averaged to mean values. 
The values of the MEPs were widely distributed and, thus, individual mean values were transformed into a percentage of the MEP at rest (\%MEP) for each subject. Then, the individual \%MEP values were analyzed with a repeatedmeasures one-way analysis of variance (ANOVA) and a post-hoc Bonferroni correction for multiple compari-

Table 2. Changes in TMS parameters according to conditions in healthy volunteers

\begin{tabular}{|c|c|c|}
\hline & Baseline & MI only \\
\hline RMT (\%MSO) & $46.8 \pm 2.37$ & - \\
\hline $\begin{array}{l}\text { ECR NC MEP } \\
\text { AMP }(\mathrm{mV})\end{array}$ & $0.63 \pm 0.11$ & $1.12 \pm 0.27$ \\
\hline $\begin{array}{l}\text { ECR NC MEP } \\
\text { area }(\mathrm{mV} / \mathrm{ms})\end{array}$ & $3.27 \pm 0.63$ & $6.15 \pm 1.76$ \\
\hline ECR \%INH & $47.8 \pm 19.05$ & $36.84 \pm 15.73$ \\
\hline ECR \%FAC & $168.17 \pm 39.81$ & $175.57 \pm 42.57$ \\
\hline \multirow[t]{2}{*}{$\operatorname{Mmax}(\mathrm{mV})$} & $8.43 \pm 2.04$ & $8.28 \pm 2.00$ \\
\hline & Baseline & VR guided MI \\
\hline RMT (\%MSO) & $46.8 \pm 2.37$ & - \\
\hline $\begin{array}{l}\text { ECR NC MEP } \\
\text { AMP }(\mathrm{mV})\end{array}$ & $0.61 \pm 0.15$ & $1.43 \pm 0.46$ \\
\hline $\begin{array}{l}\text { ECR NC MEP } \\
\text { area }(\mathrm{mV} / \mathrm{ms})\end{array}$ & $3.19 \pm 0.75$ & $7.42 \pm 2.83$ \\
\hline ECR \%INH & $44.16 \pm 15.03$ & $34.89 \pm 13.48$ \\
\hline ECR \%FAC & $162.62 \pm 40.92$ & $173.87 \pm 37.47$ \\
\hline \multirow[t]{2}{*}{$\operatorname{Mmax}(\mathrm{mV})$} & $8.43 \pm 2.04$ & $8.33 \pm 2.09$ \\
\hline & Baseline & $\begin{array}{l}\text { VR guided MI with } \\
\text { task variability }\end{array}$ \\
\hline RMT (\%MSO) & $46.8 \pm 2.37$ & - \\
\hline $\begin{array}{l}\text { ECR NC MEP } \\
\text { AMP }(\mathrm{mV})\end{array}$ & $0.58 \pm 0.10$ & $1.49 \pm 0.49$ \\
\hline $\begin{array}{l}\text { ECR NC MEP } \\
\text { area }(\mathrm{mV} / \mathrm{ms})\end{array}$ & $3.14 \pm 0.57$ & $8.28 \pm 3.51$ \\
\hline ECR \%INH & $46.78 \pm 13.58$ & $26.84 \pm 13.51$ \\
\hline ECR \%FAC & $165.2 \pm 38.21$ & $173.81 \pm 50.09$ \\
\hline $\operatorname{Mmax}(\mathrm{mV})$ & $8.43 \pm 2.04$ & $8.26 \pm 2.04$ \\
\hline
\end{tabular}

Values are represented as mean \pm standard deviation.

TMS, transcranial magnetic stimulation; MI, motor imagery; RMT, resting motor threshold; MSO, maximum stimulator output; ECR, extensor carpi radials; NC MEP, non-conditioned motor-evoked potential; \%INH, percent inhibition (Note: higher values indicate greater inhibition); \%FAC, percent facilitation (Note: higher values indicate greater facilitation); Mmax, maximum M-wave; VR, virtual reality. sons. Independent-sample t-tests were used to compare the mean values for MEP amplitude, MEP area, ICI, and ICF between the healthy group and the stroke group. All data were analyzed using SPSS software ver. 22.0 (SPSS Inc., Chicago, IL, USA).

Table 3. Changes in TMS parameters according to conditions in stroke patients

\begin{tabular}{|c|c|c|}
\hline & Baseline & MI only \\
\hline RMT (\%MSO) & $53.27 \pm 4.48$ & - \\
\hline $\begin{array}{l}\text { ECR NC MEP } \\
\text { AMP }(\mathrm{mV})\end{array}$ & $0.47 \pm 0.17$ & $0.73 \pm 0.26$ \\
\hline $\begin{array}{l}\text { ECR NC MEP } \\
\text { area }(\mathrm{mV} / \mathrm{ms})\end{array}$ & $2.49 \pm 0.89$ & $4.42 \pm 2.41$ \\
\hline ECR \%INH & $39.57 \pm 4.62$ & $31.70 \pm 4.59$ \\
\hline ECR \%FAC & $152.36 \pm 20.48$ & $159.81 \pm 26.06$ \\
\hline \multirow[t]{2}{*}{$\operatorname{Mmax}(\mathrm{mV})$} & $8.67 \pm 1.31$ & $8.68 \pm 1.26$ \\
\hline & Baseline & VR-guided MI \\
\hline RMT (\%MSO) & $53.27 \pm 4.48$ & - \\
\hline $\begin{array}{l}\text { ECR NC MEP } \\
\text { AMP }(\mathrm{mV})\end{array}$ & $0.48 \pm 0.18$ & $0.88 \pm 0.31$ \\
\hline $\begin{array}{l}\text { ECR NC MEP } \\
\text { area }(\mathrm{mV} / \mathrm{ms})\end{array}$ & $2.56 \pm 0.84$ & $5.07 \pm 2.65$ \\
\hline ECR \%INH & $40.91 \pm 4.12$ & $29.52 \pm 3.10$ \\
\hline ECR \%FAC & $145.36 \pm 20.17$ & $165.00 \pm 26.93$ \\
\hline \multirow[t]{2}{*}{$\operatorname{Mmax}(\mathrm{mV})$} & $8.67 \pm 1.31$ & $8.63 \pm 1.25$ \\
\hline & Baseline & $\begin{array}{l}\text { VR guided MI with } \\
\text { task variability }\end{array}$ \\
\hline RMT (\%MSO) & $53.27 \pm 4.48$ & - \\
\hline $\begin{array}{l}\text { ECR NC MEP } \\
\text { AMP }(\mathrm{mV})\end{array}$ & $0.48 \pm 0.17$ & $0.91 \pm 0.31$ \\
\hline $\begin{array}{l}\text { ECR NC MEP } \\
\text { area }(\mathrm{mV} / \mathrm{ms})\end{array}$ & $2.53 \pm 0.84$ & $5.05 \pm 2.67$ \\
\hline ECR \%INH & $38.55 \pm 6.38$ & $24.64 \pm 4.17$ \\
\hline ECR \%FAC & $150.34 \pm 25.65$ & $180.58 \pm 24.08$ \\
\hline $\operatorname{Mmax}(\mathrm{mV})$ & $8.67 \pm 1.31$ & $8.54 \pm 1.24$ \\
\hline
\end{tabular}

Values are represented as mean \pm standard deviation.

TMS, transcranial magnetic stimulation; MI, motor imagery; RMT, resting motor threshold; MSO, maximum stimulator output; ECR, extensor carpi radials; NC MEP, non-conditioned motor-evoked potential; \% INH, percent inhibition (Note: higher values indicate greater inhibition); \%FAC, percent facilitation (Note: higher values indicate greater facilitation); Mmax, maximum M-wave; VR, virtual reality. 


\section{RESULTS}

A comparison of the increases in \%MEP amplitudes at rest across the three testing conditions revealed a pattern of significant differences between the healthy volunteers and the stroke patients (Tables 2,3). Additionally, the MEP amplitudes $(\mathrm{p}<0.001)$ and MEP areas $(\mathrm{p}<0.001)$ were larger in healthy volunteers than in the stroke patients at rest.

\section{Changes in the MEP parameters of healthy volunteers}

In healthy volunteers, a comparison of the increases in \%MEP amplitudes across the three testing conditions revealed a pattern of significant differences $\left(\mathrm{F}_{2,28}=4.71\right.$, $\mathrm{p}=0.005)$. The increases in \%MEP amplitudes were higher under the VR-guided MI condition ( $p=0.013$ ) (Fig. 3A) and under the condition involving VR-guided MI with task variability $(\mathrm{p}=0.048)$ (Fig. $3 \mathrm{~A})$ than during $\mathrm{MI}$ alone. After each condition, the value of ECR \%INH was lowest under the condition involving VR-guided MI with task variability $(26.84 \% \pm 13.51 \%)$ than under those involving VR-guided MI $(34.89 \% \pm 13.48 \%)$ and MI alone $(36.84 \% \pm 15.73 \%)$, and the reduction in \%INH was greater under the condition involving VR-guided MI with task variability than under that involving VR-guided MI $(p=0.029)$ (Fig. 3B). The MEP area and Mmax (peripheral excitability) did not show any significant changes across
Conditions $\mathrm{B}, \mathrm{C}$, and $\mathrm{D}(\mathrm{p}=0.088$ and $\mathrm{p}=0.28$, respectively).

\section{Changes in the MEP parameters of stroke patients}

In stroke patients, a comparison of the increases in \%MEP amplitudes across the three testing conditions revealed a pattern of significant differences $\left(\mathrm{F}_{2,28}=8.581\right.$, $\mathrm{p}=0.001)$. The percentage increases in MEP amplitudes were greater during the VR-guided MI condition $(\mathrm{p}=0.009)$ and the condition involving VR-guided MI with task variability ( $\mathrm{p}=0.037$ ) than during MI alone (Fig. $4 \mathrm{~A}$ ). The increases in \%MEP area across the three testing conditions also revealed a pattern of significant differences $\left(\mathrm{F}_{2,28}=8.36, \mathrm{p}=0.001\right)$. The increases in \%MEP area were greater during the VR-guided MI condition $(\mathrm{p}=0.025)$ and the condition involving VR-guided MI with task variability $(\mathrm{p}=0.018)$ compared with that involving MI alone (Fig. 4B). After each condition, the value of ECR \%INH was lowest under the condition involving VR-guided MI with task variability $(24.64 \% \pm 4.17 \%)$ than under the VR-guided MI condition $(29.52 \% \pm 3.10 \%)$ and MI-alone condition $(31.70 \% \pm 4.59 \%)$. The reduction in \%INH was greater under the condition involving VR-guided MI with task variability than under the VR-guided MI condition ( $\mathrm{p}=0.003$ ) (Fig. 4C). The Mmax (peripheral nerve excitability) and \%FAC did not show any significant changes across Conditions $\mathrm{B}, \mathrm{C}$, and $\mathrm{D}$ ( $\mathrm{p}=0.195$ and $\mathrm{p}=0.082$, respectively).
(A)

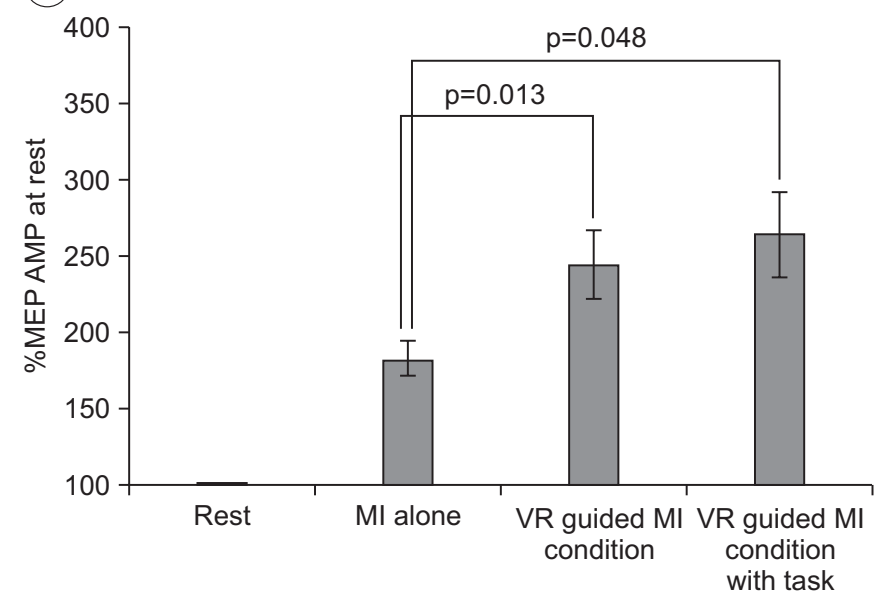

(B)

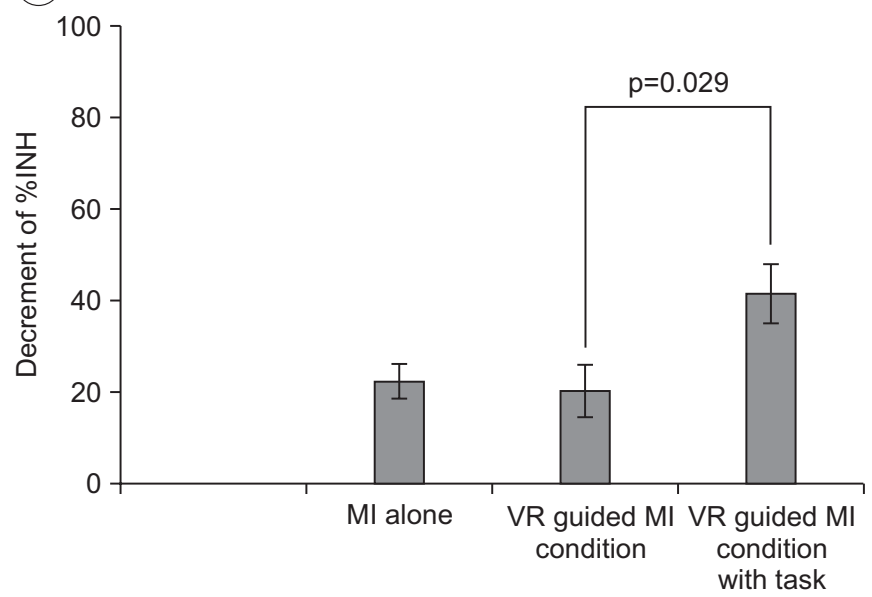

Fig. 3. Changes in the transcranial magnetic stimulation parameters of healthy volunteers. (A) The \%MEP amplitude was significantly greater under the VR-guided MI conditions than under the MI alone condition. (B) The reduction in \%INH was greater under the VR-guided MI condition with task variability than under that with regular intervals. Values are expressed as mean \pm standard error of mean. MEPs, motor-evoked potentials; VR, virtual reality; MI, motor imagery; \%INH, percent inhibition. 


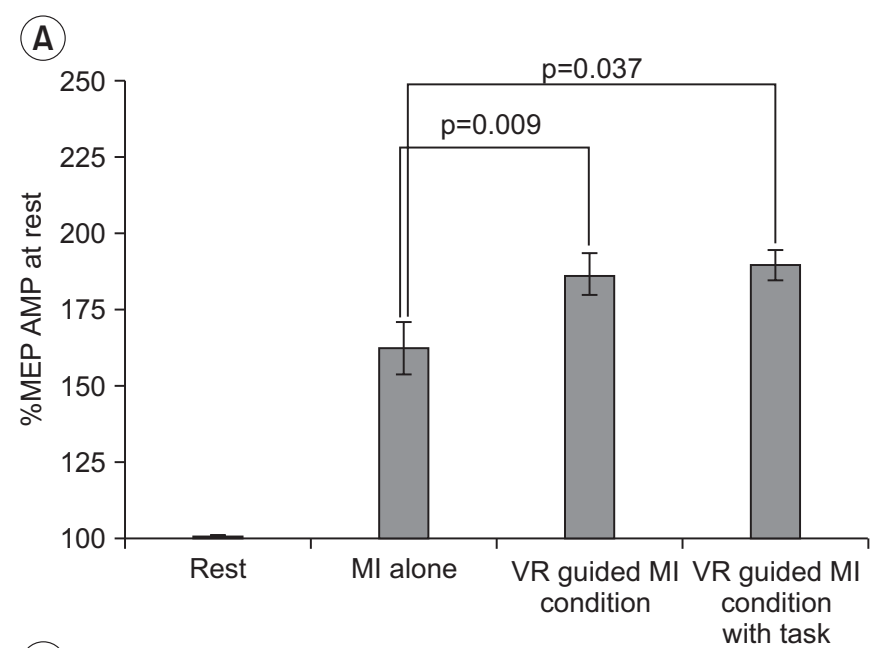

(C)

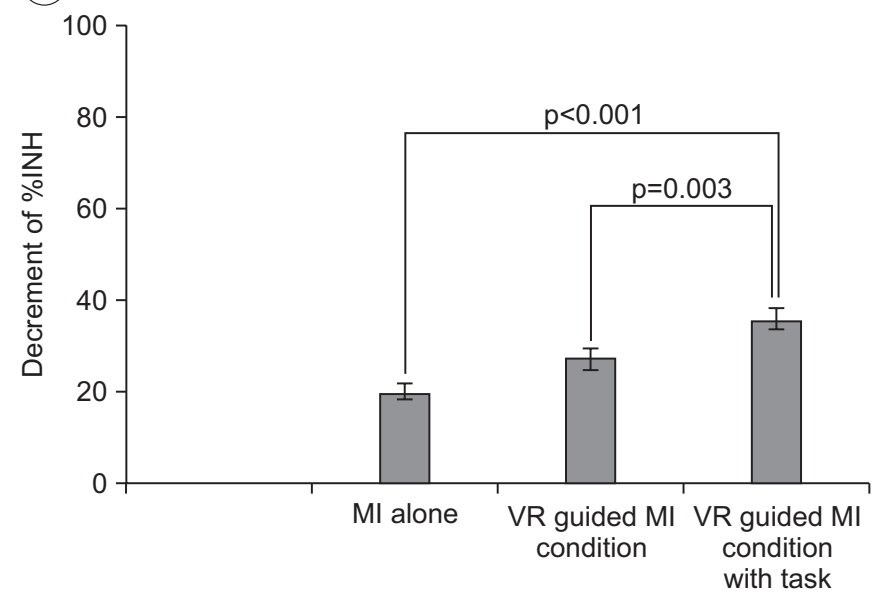

\section{Adverse effects}

There were no significant adverse effects during either the TMS or VR procedures, and only a few mild adverse events were reported in general: a mild headache (in one stroke patient) and neck pain (in one stroke patient and two healthy volunteers).

\section{VMIO-2 scores}

The VMIQ-2 scores indicated that all subjects had reasonably clear and vivid images when bringing images to mind; the mean scores per item were $1.95 \pm 0.22$ (range, $1.67-2.25$ ) for the stroke patients and $1.84 \pm 0.18$ (range, $1.58-2.25)$ for the healthy volunteers. Thus, all subjects were able to form clear and reasonably vivid images from an MI perspective.

\section{DISCUSSION}

In the present study, the increases in \%MEP ampli-

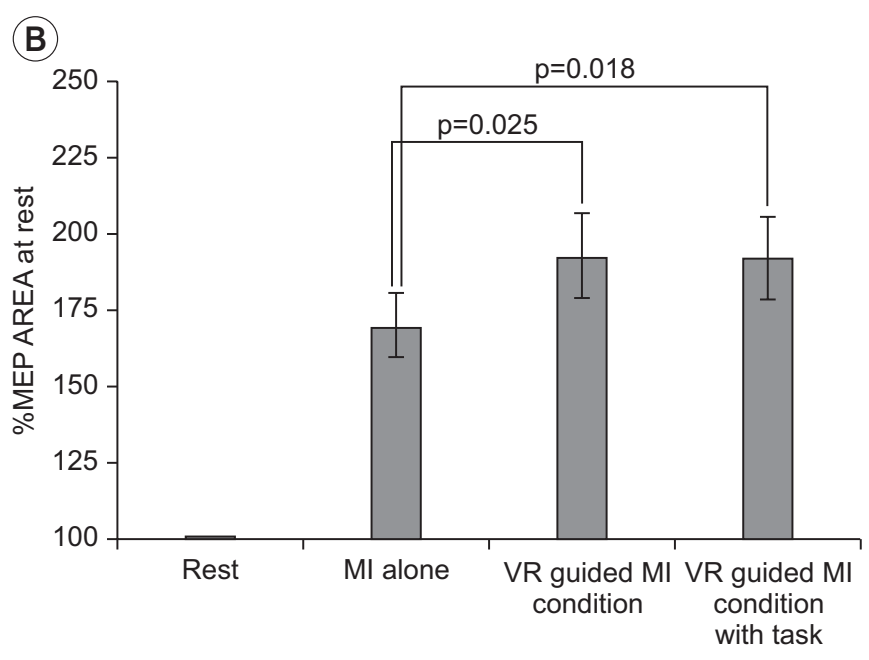

Fig. 4. Changes in the transcranial magnetic stimulation parameters of stroke patients. (A) The increase in \%MEP amplitude was significantly greater under the VR-guided MI conditions than under the MI alone condition. (B) The VR-guided MI conditions resulted in a significant increase in \%MEP area compared with MI alone. (C) The reduction in \%INH was greater under the VR-guided MI condition with task variability than under that with regular intervals. Values are expressed as mean \pm standard error of mean. MEPs, motor-evoked potentials; VR, virtual reality; MI, motor imagery; \%INH, percent inhibition.

tude were greater in both the healthy volunteers and the stroke patients under the VR-guided MI conditions, regardless of the presence of task variability, as compared with the MI-alone condition. For both groups, the \%INH values after each condition were lowest under the condition involving VR-guided MI with task variability, and the reduction in \%INH was greater under the VR-guided MI condition with task variability than under the VR-guided MI condition with regular intervals.

\section{MI-induced increases in cortical excitability}

Although cortical excitation during MI may be weaker than during actual movement, a number of previous studies have reported that MI-induced corticospinal facilitation occurs in both healthy volunteers and stroke patients [19]. In present study, to allow for maximal facilitation, we assessed kinesthetic MI while observing his/her real body or VR program. Larger MEP amplitudes are observed during combination of MI and action ob- 
servation [20] and during the kinesthetic imagination of movements than the visual imagination of movements [21]. The basic mechanisms underlying MI are thought to be associated with the activation of the mirror neuron system, an extended motor network in the cerebral cortex that is activated during the execution of motor imagery [22]. fMRI studies have shown that the regional activation patterns in this extended motor network are similar in stroke patients and healthy individuals during MI [23]. In this study, the healthy volunteers and the stroke patients exhibited similar corticomotor excitability changes during and after MI. Our findings are consistent with those of a previous study that demonstrated similar facilitation patterns in mildly impaired stroke patients and healthy individuals during MI [24].

Peripheral nerve excitability was assessed by evaluating changes in the Mmax of the median nerve of the wrist, which did not change after any of the conditions. Previous studies demonstrated that little or no change of Fwave is elicited by peripheral nerve stimulation during MI $[21,25]$. Thus, it is likely that the marked facilitation of MEP amplitude we observed involved the cortical level rather than the spinal or peripheral levels.

\section{A combination of MI and VR increases cortical excita- bility}

The present findings demonstrated that the combined effects of MI and VR training were synergistic and that the corticomotor facilitation produced by this combination was superior to that produced by MI alone among both stroke patients and healthy volunteers.

The issue of whether there are differences in brain activation during real versus virtual action remains controversial. A positron emission tomography (PET) study found that the activation of the mirror neuron system occurs during the observation of a real-body grasping task but not in an artificial-arm task [26]. However, an fMRI study showed that the mirror neuron system is strongly activated by the sight of both human and virtual (robotic) actions, with no significant differences between the two conditions [27]. This suggests that the goal rather than the manner in which the action is performed may be more important for cortical activation. Recent research has shown that corticospinal excitability is increased during a goal-directed VR mirror exercise relative to a real mirror exercise [28]. In addition, action related sound of
VR associated with jumping might facilitate the corticospinal excitability [29].

In a situation immersed in a virtual environment, a controller that could manipulate or control movement (wrist extension in this program) should be provided. We designed a character (avatar) running and jumping over the obstacles to evoke user's attention and hypothesized that it induced more efficient goal-directed MI ability than those inducing an internal focus by observation of the real body movements (wrist extension) themselves. Therefore, we aimed to investigate the effect of corticomotor facilitation according to traditional MI (real body observation) and VR-guided MI (VR avatar representation). The present findings are important because they support the use of various goal-directed avatar-based audiovisual VR programs and the concept of combining MI with VR for neurorehabilitation in stroke patients.

In the present study, the increases in MEP amplitude and area and the reductions in \%INH in stroke patients were greater under the VR-guided MI conditions than under MI alone. Increased corticospinal excitability and reduced ICI are essential for the induction of neural plasticity following brain injury in animal studies [30,31]. Previous studies have shown that MI increased corticospinal excitability and decreased ICI and that MI training induced motor cortical plasticity [32,33]. These findings suggest that the increased intracortical circuit responsiveness during VR-guided MI might induce the neural reorganization after stroke. A Cochrane review reported beneficial effects on patients who received more training in MI or VR, as compared with patients who received less training [2]; thus, further clinical studies are needed to determine the optimal amount of MI-VR training for stroke patients.

Moreover, a previous study found that MI and VR are promising therapeutic strategies for balance and gait disorders, such as Parkinson disease, because these patients exhibit improved balance and gait following treatment [34].

\section{Task variability under the MI-VR condition decrease ICI}

In the present study, VR-guided MI with task variability significantly reduced the ICI in both the healthy volunteers and stroke patients. Additionally, the \%INH after each condition was lowest under the VR-guided MI with task variability, as compared with the VR-guided 
MI regular intervals. A few studies have assessed the task dependent changes in ICI during MI. Stinear and Byblow [33] reported that ICI was modulation by imagery of muscle contraction task and not by simple phasic task. Several studies have assessed increase in MEP amplitude in the presence of task variability or the level of difficulty during MI or VR motor training $[28,35]$. In this study, the change of MEP amplitude and area were not statistically significant according to task variability of VR; hence, further study is needed. In addition, the change of ICF according to task variability was not significant in this study. Previous study supported the idea that ICI and ICF may be controlled independently [36]. The induction of effective motor learning is highly dependent on task variability or adjustments in the level of difficulty [28,37], and these task characteristics are easily modulated in a virtual environment. Therefore, the present findings support the idea that different VR programs that include task variability might effectively modulate the cortico-cortical inhibitory circuit and ultimately influence neural plasticity.

The present study has several limitations that need to be considered. First, this study had a relatively small sample of stroke patients with mild-to-moderate impairments. Patients with a severe motor weakness (MRC grade of wrist extension $\leq 2$ ) were excluded because this population typically does not exhibit MEPs in the effected ECR muscle. Second, the heterogeneous nature of the lesions (cortical vs. subcortical), the varied times of onset, and the chosen side of stimulation may have also influenced the results of the present study. Third, because MI is an individual mental process, it was difficult to objectively assess the MI ability of each subject. Recent advances in brain-computer interfaces have made it easier to use electroencephalogram findings, specifically event-related desynchronization or synchronization, as objective assessments of MI performance [38]. Fourth, we did not assess ICI, ICF and peripheral nerve excitability during MI because of methodological difficulties. The present study revealed that ICI was decreased after MI and VR and MI alone condition. Our finding is congruence with the previous work of Classen et al. [39] who reported that corticomuscular excitability of muscle lasted at least 10 minutes after physical training and observational training also produced similar change. We found that ICI decreased immediately after the MI or VR and MI conditions, which supports the findings of Zhang et al. [40], who reported that resting-state functional connectivity could be modulated by MI.

Our findings demonstrated that corticomotor excitability is modulated during and after VR-guided MI training; thus, it is reasonable to consider that this combination of techniques is a promising treatment option in the field of neurorehabilitation. To use VR-guided MI training as a therapeutic strategy, corticomotor excitability has to be maintained. Further study could be conducted to investigate the electrophysiological and clinical therapeutic effect of VR-guided MI after repetitive task training using various subgroups of stroke patients, different training methods, and varied durations of training.

In conclusion, the corticomotor excitability elicited by MI using VR avatar representations was greater than that elicited during MI alone using real body observations. Furthermore, task variability within a VR program may enhance neural regeneration after stroke by reducing ICI. These neurophysiological data support the application of various VR programs as well as the concept of combining MI with VR programs for the neurorehabilitation of stroke patients.

\section{CONFLICT OF INTEREST}

No potential conflict of interest relevant to this article was reported.

\section{ACKNOWLEDGMENTS}

We thank Hye Sun Lee at the Department of MedicalStatistics in Yonsei University of College of Medicine for valuable comments on statistical analyses of the results. This study was supported by the Basic Science Research Program through the National Research Foundation of Korea (NRF) funded by the Ministry of Education (No. 2013R1A1A2060973).

\section{REFERENCES}

1. Dobkin BH. Clinical practice. Rehabilitation after stroke. N Engl J Med 2005;352:1677-84.

2. Pollock A, Farmer SE, Brady MC, Langhorne P, Mead GE, Mehrholz J, et al. Interventions for improving upper limb function after stroke. Cochrane Database 


\section{Syst Rev 2014;11:CD010820.}

3. Mulder T. Motor imagery and action observation: cognitive tools for rehabilitation. J Neural Transm (Vienna) 2007;114:1265-78.

4. Lotze M, Montoya P, Erb M, Hulsmann E, Flor H, Klose U, et al. Activation of cortical and cerebellar motor areas during executed and imagined hand movements: an fMRI study. J Cogn Neurosci 1999;11:491501.

5. Kho AY, Liu KP, Chung RC. Meta-analysis on the effect of mental imagery on motor recovery of the hemiplegic upper extremity function. Aust Occup Ther J 2014;61:38-48.

6. Subramanian SK, Lourenco CB, Chilingaryan G, Sveistrup H, Levin MF. Arm motor recovery using a virtual reality intervention in chronic stroke: randomized control trial. Neurorehabil Neural Repair 2013;27:1323.

7. Turolla A, Dam M, Ventura L, Tonin P, Agostini M, Zucconi C, et al. Virtual reality for the rehabilitation of the upper limb motor function after stroke: a prospective controlled trial. J Neuroeng Rehabil 2013;10:85.

8. Prochnow D, Bermudez i Badia S, Schmidt J, Duff A, Brunheim S, Kleiser R, et al. A functional magnetic resonance imaging study of visuomotor processing in a virtual reality-based paradigm: rehabilitation gaming system. Eur J Neurosci 2013;37:1441-7.

9. Diers M, Kamping S, Kirsch P, Rance M, BekraterBodmann R, Foell J, et al. Illusion-related brain activations: a new virtual reality mirror box system for use during functional magnetic resonance imaging. Brain Res 2015;1594:173-82.

10. Brunner I, Skouen JS, Hofstad H, Strand LI, Becker F, Sanders AM, et al. Virtual reality training for upper extremity in subacute stroke (VIRTUES): study protocol for a randomized controlled multicenter trial. BMC Neurol 2014;14:186.

11. Fluet GG, Deutsch JE. Virtual reality for sensorimotor rehabilitation post-stroke: the promise and current state of the field. Curr Phys Med Rehabil Rep 2013;1:920.

12. Cicinelli P, Pasqualetti P, Zaccagnini M, Traversa R, Oliveri M, Rossini PM. Interhemispheric asymmetries of motor cortex excitability in the postacute stroke stage: a paired-pulse transcranial magnetic stimulation study. Stroke 2003;34:2653-8.
13. Liepert J, Storch P, Fritsch A, Weiller C. Motor cortex disinhibition in acute stroke. Clin Neurophysiol 2000;111:671-6.

14. Leonard G, Tremblay F. Corticomotor facilitation associated with observation, imagery and imitation of hand actions: a comparative study in young and old adults. Exp Brain Res 2007;177:167-75.

15. Oldfield RC. The assessment and analysis of handedness: the Edinburgh inventory. Neuropsychologia 1971;9:97-113.

16. Rossi S, Hallett M, Rossini PM, Pascual-Leone A; Safety of TMS Consensus Group. Safety, ethical considerations, and application guidelines for the use of transcranial magnetic stimulation in clinical practice and research. Clin Neurophysiol 2009;120:2008-39.

17. Folstein MF, Folstein SE, McHugh PR. "Mini-mental state". A practical method for grading the cognitive state of patients for the clinician. J Psychiatr Res 1975;12:189-98.

18. Roberts R, Callow N, Hardy L, Markland D, Bringer J. Movement imagery ability: development and assessment of a revised version of the vividness of movement imagery questionnaire. J Sport Exerc Psychol 2008;30:200-21.

19. Hashimoto R, Rothwell JC. Dynamic changes in corticospinal excitability during motor imagery. Exp Brain Res 1999;125:75-81.

20. Naish KR, Houston-Price C, Bremner AJ, Holmes NP. Effects of action observation on corticospinal excitability: muscle specificity, direction, and timing of the mirror response. Neuropsychologia 2014;64C:331-48.

21. Stinear CM, Byblow WD, Steyvers M, Levin O, Swinnen SP. Kinesthetic, but not visual, motor imagery modulates corticomotor excitability. Exp Brain Res 2006;168:157-64.

22. Buccino G, Solodkin A, Small SL. Functions of the mirror neuron system: implications for neurorehabilitation. Cogn Behav Neurol 2006;19:55-63.

23. Sharma N, Baron JC, Rowe JB. Motor imagery after stroke: relating outcome to motor network connectivity. Ann Neurol 2009;66:604-16.

24. Kang YJ, Ku J, Kim HJ, Park HK. Facilitation of corticospinal excitability according to motor imagery and mirror therapy in healthy subjects and stroke patients. Ann Rehabil Med 2011;35:747-58.

25. Rossini PM, Rossi S, Pasqualetti P, Tecchio F. Cortico- 
spinal excitability modulation to hand muscles during movement imagery. Cereb Cortex 1999;9:161-7.

26. Tai YF, Scherfler C, Brooks DJ, Sawamoto N, Castiello $\mathrm{U}$. The human premotor cortex is 'mirror' only for biological actions. Curr Biol 2004;14:117-20.

27. Gazzola V, Rizzolatti G, Wicker B, Keysers C. The anthropomorphic brain: the mirror neuron system responds to human and robotic actions. Neuroimage 2007;35:1674-84.

28. Kang YJ, Park HK, Kim HJ, Lim T, Ku J, Cho S, et al. Upper extremity rehabilitation of stroke: facilitation of corticospinal excitability using virtual mirror paradigm. J Neuroeng Rehabil 2012;9:71.

29. Aziz-Zadeh L, Iacoboni M, Zaidel E, Wilson S, Mazziotta J. Left hemisphere motor facilitation in response to manual action sounds. Eur J Neurosci 2004;19:260912.

30. Ljubisavljevic M. Transcranial magnetic stimulation and the motor learning-associated cortical plasticity. Exp Brain Res 2006;173:215-22.

31. Jacobs KM, Donoghue JP. Reshaping the cortical motor map by unmasking latent intracortical connections. Science 1991;251:944-7.

32. Avanzino L, Gueugneau N, Bisio A, Ruggeri P, Papaxanthis C, Bove M. Motor cortical plasticity induced by motor learning through mental practice. Front Behav Neurosci 2015;9:105.

33. Stinear CM, Byblow WD. Modulation of corticospinal excitability and intracortical inhibition during mo- tor imagery is task-dependent. Exp Brain Res 2004; 157:351-8.

34. Mirelman A, Maidan I, Deutsch JE. Virtual reality and motor imagery: promising tools for assessment and therapy in Parkinson's disease. Mov Disord 2013;28:1597-608.

35. Roosink M, Zijdewind I. Corticospinal excitability during observation and imagery of simple and complex hand tasks: implications for motor rehabilitation. Behav Brain Res 2010;213:35-41.

36. Liepert J, Classen J, Cohen LG, Hallett M. Task-dependent changes of intracortical inhibition. Exp Brain Res 1998;118:421-6.

37. Krakauer JW. Motor learning: its relevance to stroke recovery and neurorehabilitation. Curr Opin Neurol 2006;19:84-90.

38. Ang KK, Guan C, Chua KS, Ang BT, Kuah CW, Wang C, et al. A large clinical study on the ability of stroke patients to use an EEG-based motor imagery braincomputer interface. Clin EEG Neurosci 2011;42:253-8.

39. Classen J, Liepert J, Wise SP, Hallett M, Cohen LG. Rapid plasticity of human cortical movement representation induced by practice. J Neurophysiol 1998;79: 1117-23.

40. Zhang H, Long Z, Ge R, Xu L, Jin Z, Yao L, et al. Motor imagery learning modulates functional connectivity of multiple brain systems in resting state. PLoS One 2014;9:e85489. 